\title{
A Vision for Online Verification-Validation
}

\author{
Matthew A. Hammer \\ University of Colorado Boulder, USA \\ matthew.hammer@colorado.edu
}

\author{
Bor-Yuh Evan Chang \\ University of Colorado Boulder, USA \\ evan.chang@colorado.edu
}

\author{
David Van Horn \\ University of Maryland, \\ College Park, USA \\ dvanhorn@cs.umd.edu
}

\begin{abstract}
Today's programmers face a false choice between creating software that is extensible and software that is correct. Specifically, dynamic languages permit software that is richly extensible (via dynamic code loading, dynamic object extension, and various forms of reflection), and today's programmers exploit this flexibility to "bring their own language features" to enrich extensible languages (e.g., by using common JavaScript libraries). Meanwhile, such librarybased language extensions generally lack enforcement of their abstractions, leading to programming errors that are complex to avoid and predict.

To offer verification for this extensible world, we propose online verification-validation (OVV), which consists of language and VM design that enables a "phaseless" approach to program analysis, in contrast to the standard static-dynamic phase distinction. Phaseless analysis freely interposes abstract interpretation with concrete execution, allowing analyses to use dynamic (concrete) information to prove universal (abstract) properties about future execution.

In this paper, we present a conceptual overview of OVV through a motivating example program that uses a hypothetical database library. We present a generic semantics for $\mathrm{OVV}$, and an extension to this semantics that offers a simple gradual type system for the database library primitives. The result of instantiating this gradual type system in an OVV setting is a checker that can progressively type successive continuations of the program until a continuation is fully verified. To evaluate the proposed vision of OVV for this example, we implement the VM semantics (in Rust), and show that this design permits progressive typing in this manner.
\end{abstract}

Categories and Subject Descriptors $\quad$ D.3.1 [Programming Languages]: Formal Definitions and Theory; D.3.3 [Pro-

Permission to make digital or hard copies of all or part of this work for personal or classroom use is granted without fee provided that copies are not made or distributed for profit or commercial advantage and that copies bear this notice and the full citation on the first page. Copyrights for components of this work owned by others than ACM must be honored. Abstracting with credit is permitted. To copy otherwise, or republish, to post on servers or to redistribute to lists, requires prior specific permission and/or a fee. Request permissions from Permissions@acm.org.

GPCE'16, October 31 - November 1, 2016, Amsterdam, Netherlands (c) 2016 ACM. 978-1-4503-4446-3/16/10...\$15.00

http://dx.doi.org/10.1145/2993236.2993255 gramming Languages]: Language Constructs and Features; F.3.2 [Logics and Meanings of Programs]: Semantics of Programming Languages

Keywords Online Verification-Validation, Virtual Machines, For-all Analysis, Exists Analysis, Phaseless Analysis, Type Systems, Call-by-Push-Value (CBPV).

\section{Introduction}

We consider the problem of typing unknown, dynamicallydetermined data obtained from the environment. To illustrate what we mean by unknown, dynamically-determined data, consider the code in Figure 1a that loads a comma-separated value (CSV) file authors. csv using the openDb ("authors.csv") call on line 1. The contents of this file are organized into lines, where each line is a row of field values. The first line of the file is special and contains a list of field names instead of field value-see Figure $1 \mathrm{~b}$ for example content. In line 1 of Figure 1a, the programmer filters the author list down to those that have US citizenship using the field projection author.citizenship.

Suppose the programmer merely wants to know that their code will not access undefined fields-that, in this respect, the program is well-typed. Given that these object fields are defined by the dynamic-generation of data structures via openDb, the validity of this field projection author.citizenship is generally unknowable until after line 1 when the structure of the authors table is defined based on the contents of authors.csv. At the same time, this field projection is clearly valid because of the special first line in specifically this authors.csv shown in Figure $1 \mathrm{~b}$.

There is a tension here between static and dynamic checking. On one hand, once the authors table has been determined, the programmer would like the field projections from the rows to be statically verified. But on other hand, how would static checking be feasible if the schema of authors database is dynamically-determined-what would be the type of the openDb function?

To resolve this tension, our key insight is to imagine pausing the concrete execution of Figure $1 \mathrm{a}$ right after line 1 and before line 2. Then, imagine reflecting the continuation of the program's execution to apply abstract interpretation or 
(a) Open a database with an implicit schema and filter the content.

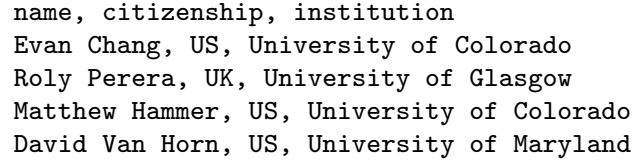

(b) File authors.csv, example content.

Figure 1. Programming with dynamically-determined tables and objects in JavaScript

other static techniques to prove the validity of all subsequent field projections on the rows of the authors table.

Our vision centers around a new paradigm for verification and validation that we call online verification-validation (OVV). In contrast to today's phasic analysis techniques, where static and dynamic checking are separated into distinct, non-overlapping phases, OVV is phaseless: "static" analysis is freely interposed with "dynamic" execution. By virtue of this mixed approach, OVV transcends the conventional phase distinctions of "static" and "dynamic" analysis. To avoid confusion in this phaseless setting, we refer to static analysis techniques as those for $\forall$-analysis, since they demonstrate universal properties of future program's states. Likewise, we refer to the techniques used in dynamic analysis as those for $\exists$-analysis, since they demonstrate existential properties about the past program's states.

In this paper, we make the following contributions:

- We define $\lambda$-VMF, an abstract machine semantics that enables a form of phaseless, online verification-validation (Section 3). The key idea is annotating potentially faulting operations (e.g., field projection) with a certain (!) or uncertain (?) flag, indicating whether or not that operation can be verified. Executing uncertain operations gets stuck, so it is up to the OVV program to progressively rewrite uncertain operations into certain ones by proving the safety of the potentially faulting operation.

- We present a case study of instantiating online verification-validation with a simple, bidirectional gradual type system for dynamic field projection and databases with dynamic schemas (Section 4). The result of instantiating a gradual type system in an online verification-validation setting is a checker that can progressively type successive continuations of the program until a continuation is fully verified (i.e., "statically" typed). ${ }^{1}$

- We implement the proposed design for $\lambda$-VMF in Rust to demonstrate that the proposed instantiation of OVV

\footnotetext{
${ }^{1} \mathrm{~A}$ continuation of a running program represents its state, including the remainder of the code to be executed in the future.
}

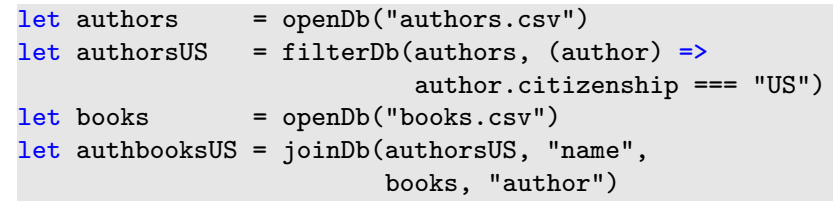

(a) The joinDb (db1, key1, db2, key2) library function accesses the key fields using run-time reflection (e.g., db1 [i] [key1]).

author, title, year, publisher

...

(b) File books.csv, example content.

Figure 2. Continuing the example from Figure 1 a with subsequent dynamically-determined data.

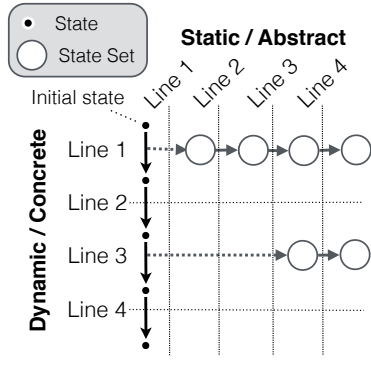

(a) Phaseless, online verificationvalidation. (b) Phasic, offline verification and online validation

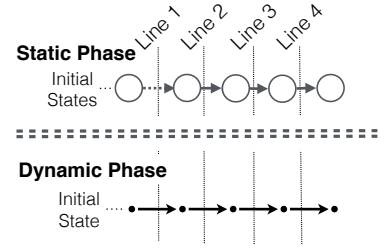

Figure 3. Typical type checkers are phasic with an offline static verifier and an online dynamic validator.

indeed realizes progressive typing. Our implementation is public: https://github.com/cuplv/vmfuture

In the next section, we dig deeper into what we term online verification-validation by following the progressive typing of the example program from Figure $1 \mathrm{a}$.

\section{Overview}

In Figure 2a consider an extension of the example from Figure 1a with two additional lines. The file books.csv is opened on line 3 and contains a CSV file with book information (shown in Figure 2b). The final line (line 4) creates a table of books written by US authors, along with the information about those authors.

Observe that this code alternates between dynamically determining the types of the rows and tables (by reading the two files authors.csv and books.csv on lines 1 and 3 and computing over those tables (on lines 2 and 4). This example is a simple version of a pervasive pattern in dynamic languages, where execution interleaves dynamic steps that create new data types (the tables loaded in lines 1 and 3) and steps that compute over previously-defined data types (the filtering and joining steps in lines 2 and 4 .

Phaseless Analysis. The essence of online verificationvalidation is pausing concrete execution to interleave it with 
abstract interpretation. Pictorially, this interleaving of concrete and abstract execution can be visualized as a twodimensional grid, as in Figure 3a. The vertical axis represents the extent of concrete execution and dynamic, $\exists-$ analysis (measured by program line), and for each such point, the horizontal axis represents the extent of abstract interpretation and static, $\forall$-analysis performed at this dynamic execution point. As shown, abstract interpretation explores the states after lines 2-4 after pausing at line 1, but revisits the state after line 4 again after pausing at line 3 . That is, we imagine suspending the concrete execution of the program in Figure $2 \mathrm{a}$ after the openDb call on line 1 and then interpreting the continuation to statically compute, under an abstraction, the set of reachable future states from this suspended current state (the horizontal axis).

This online look-ahead would permit us to check the projections of author.citizenship and db1[i]["name"] (on lines 2 and 4 , respectively) before the running program executes them concretely. However, the other projection in the joinDb call on line 4 -the projection of the author field (i.e., $\mathrm{db} 2[j][$ "author"] ) - cannot be proven valid in this continuation because the books. csv database has not yet been loaded. But imagine similarly suspending the concrete execution again after line 3 (shown as the bottom horizontal execution in Figure 3a). Now in this continuation, an abstract interpretation can easily verify this last remaining projection on all future concrete executions from this point.

For exposition, this example is short, and the distance between the concrete points for static checks and the future concrete points of potential failure (a bad projection) are tiny: they only consist of one or two lines. In the general case, however, the distance between these points can be arbitrarily large. For instance, a long-running scientific workload may last days or weeks, and in these cases, it is critical to know about possible future execution failures as soon as possible, to minimize interruption due to programming errors.

Related Work: Traditional Analyses are Phasic. Typical, existing program analyses impose phase distinctions between static and dynamic steps, forcing each phase to use one approach or the other. At one extreme, today's techniques for static verification explore all possible execution paths, but have no knowledge of the dynamic execution environment. At the other extreme, dynamic validation explores one path of execution: the path determined by concrete execution.

Type system design is, in general, a tradeoff in checking in an offline, static phase (e.g., are function application expressions well-typed?) and checking in the online, dynamic phase (e.g., is an array-index in bounds?). We illustrate this phasic architecture in Figure $3 b$ where the static and dynamic phases are sequenced and independent.

Some techniques attempt to "blend" these static and dynamic phases, so that information gleaned from one phase

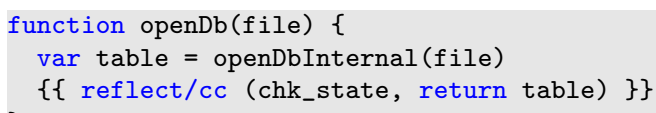

Figure 4. The openDb library function synthesizes a type for the loaded table before returning via reflect/cc.

feeds into the other. For instance, gradual typing [36, 37] enables shifting type checking between the static and dynamic phases. Or, the notion of using a set of dynamic runs to glean information before static verification appears several times in the literature for call resolution [15], reflection instantiation [9] and for eval [20, 41]. We consider such techniques phasic if there is some sequencing (rather than interleaving) of static, $\forall$-analysis and dynamic, $\exists$-analysis phases.

While phasic analyses dominate the literature, there exist some non-phasic analysis techniques, such as the "proofsfrom-tests" approach [6], that mix $\exists$-analysis information during $\forall$-analysis (e.g., via directed-random automated testing [22]). In these works, the goal is to perform offline static verification whose abstraction selection leverages $\exists$-analysis information from testing. This is distinct from our vision, where concrete execution is interposed with analyses (not vice versa). However, this work shares our concern with incremental exploration of a state space, and it possible that our proposed incremental substrate would also be beneficial in the context of "proofs-from-tests."

Online Verification-Validation. OVV programs consist of two stratified layers that interact during execution. First, the object layer expresses ordinary execution. Execution can escape into the meta layer, which is capable of inspecting the run-time representation of the object layer. Code at the meta layer expresses $\forall$-analysis and $\exists$-analysis over object programs. During ordinary execution, the meta layer plays a passive role until special primitives transfer control. In particular, reflect/cc transfers control, along with a reflected view of the current (object layer) continuation. Further, the meta layer has access to read and write hidden annotations on object layer values (e.g., to store program facts, such as types). Collectively, these hidden annotations can be viewed as providing a "shadow heap" for tracking dynamic, metalevel information, in the service of performing $\exists$-analyses. For maximum extensibility, the object layer lacks a static type system, relying on checking at the other layer. For convenience in expressing analysis over object programs, the meta layer may employ a language that employs a static type system (a la ML), but this is not a requirement. For concreteness in presentation, we use JavaScript syntax for object layer code and Rust-like syntax for meta layer code, and we may consider language choice as an orthogonal concern.

reflect/cc. The implementor of openDb uses reflect/cc to mediate between concrete execution in the object layer, and interposed code in the meta layer that performs on- 


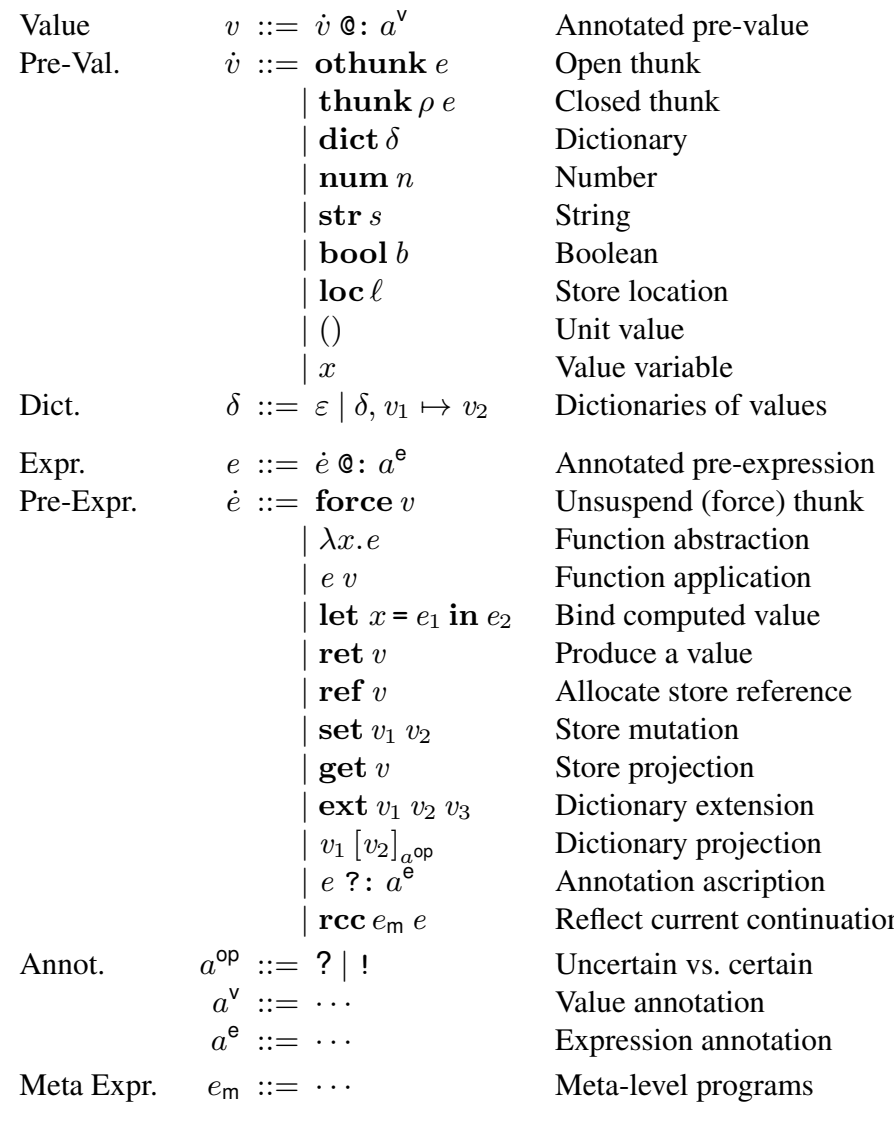

Figure 7. Syntax of $\lambda$-VMF Programs

of a database, or a record, where the typical notion of a field name is generalized to any value in $\lambda$-VMF.

Pre-expressions consist of forcing a suspended expression (force $v$ ), function abstraction $(\lambda x . e$ ), function application ( $e v$ ), let-binding a returned value (let $x=e_{1}$ in $e_{2}$ ), returning a value (ret $v$ ), allocating, mutating and accessing mutable storage (ref $v$, set $v_{1} v_{2}$, get $v$, respectively), updating the field of a record (ext $v_{1} v_{2} v_{3}$ ), projecting the field of a record $\left(v_{1}\left[v_{2}\right]_{a^{\text {op }}}\right)$.

Finally, $\lambda$-VMF includes special forms for ascribing a sub-expression with a manual annotation $\left(e ?: a^{\mathrm{e}}\right)$, and reflectively inspecting (and transforming) the current continuation via reflect/cc as core primitive, $\operatorname{rcc} e_{\mathrm{m}} e$. Notably, execution pauses before executing the local continuation $e$, and a common idiom consists of using a manual ascription there, to be discharged via the use of rcc.

As explained below, using rcc to prove and discharge ascriptions is actually necessary in $\lambda$-VMF, since they have no other form of dynamic semantics. For this purpose, the meta-level program $e_{\mathrm{m}}$ transforms the program state before its continuation resumes. We do not model the modellevel programming language here; our current implementation uses Rust.

$$
\begin{aligned}
& \text { State } \quad \sigma::=\langle\mu ; \kappa ; \rho ; \dot{e}\rangle \\
& \text { Store } \quad \mu::=\varepsilon \mid \mu, \ell \mapsto v \quad \text { Maps locations to values } \\
& \text { Stack } \quad \kappa::=\text { halt } \quad \text { Empty stack } \\
& \kappa::(\rho, x . e) \quad \text { Waiting for return } \\
& \mid \kappa:: v \quad \text { Fun. application argument } \\
& \text { Environment } \quad \rho::=\varepsilon \mid \rho, x \mapsto v \quad \text { Maps variables to values }
\end{aligned}
$$

Figure 8. VM State: The store, stack and environment.

VM State syntax. Figure 8 defines the global state of the $\lambda$-VMF program: It consists of a store, mapping locations to mutable values $(\mu)$; a stack of evaluation context frames $(\kappa)$, an environment mapping variables to values $(\rho)$, and a preexpression $\dot{e}$ that gives the current local continuation. Nonempty stacks give evaluation contexts for let bodies $(\kappa::$ $(\rho, x . e))$ and function application $(\kappa:: v)$.

Dynamics of $\lambda$-VMF Programs. Figure 9 defines a smallstep operational semantics over $\lambda$-VMF states. The rules for let and function application each push the stack with a frame that is eliminated by the rules for ret and function abstraction, respectively. In both cases, eliminating the frame consists of binding a value to a variable, and continuing the program. Forcing a thunk consists of unpacking its environment and expression, and continuing execution with them. Rules for allocating, mutating and accessing a reference cell in the store are each standard. Dictionary extension adds a field to a (possibly empty) dictionary; and dictionary projection selects a given field's associated value, returning it.

What makes $\lambda$-VMF particularly interesting is that there are no stepping rules for ?-mode field projection or for ascription. To avoid getting stuck at these operations, these operations should be either verified progressively or validated, perhaps immediately before executing, when all relevant information is available. To do so, the program uses $\operatorname{rcc} e_{\mathrm{m}} e$, which runs the meta-level program $e_{\mathrm{m}}$ on a reflected version of the current VM state: the rule constructs the current continuation, reflects this program state into a data structure, runs the meta-level term $e_{\mathrm{m}}$, and then injects the resulting program state into a transformed continuation $\sigma^{\prime}$. In OVV, this step sometimes verifies and validates operations before the program attempts to execute them, transforming the program to remove or modify them. In contrast to traditional phasic static verification or dynamic validation (cf. Figure 3b, this step does not need to be either eagerly before the entire execution or lazily just before the potentially faulting operation. By choosing the placement of rcc, the program can choose how eager or lazy the checking should be anywhere between these two extremes. We give a detailed example below.

\section{Gradual Typing for Simple Databases}

In this section we present a gradual type system for $\lambda$ VMF and libDb, an extension that permits us to express the 


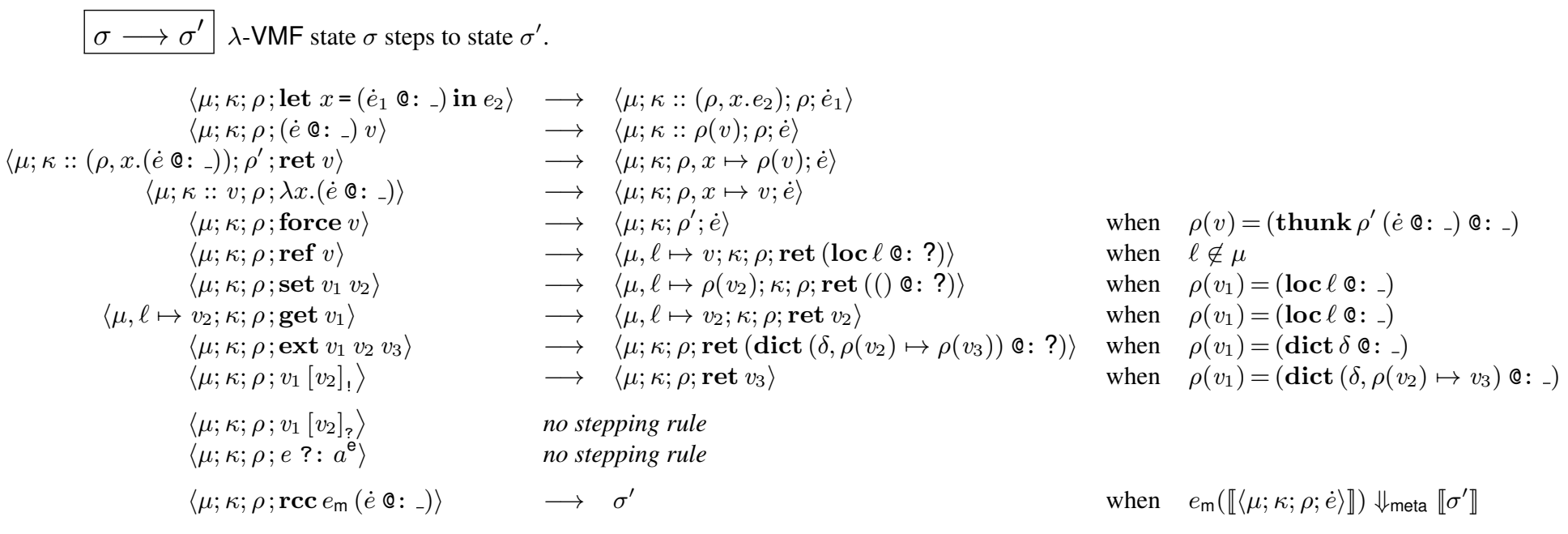

Figure 9. Small-step, abstract machine semantics of $\lambda$-VMF

\begin{abstract}
$e::=\cdots$
| openDb $a_{a \text { op }} v$

filter $\mathrm{Db}_{a}$ op $v_{1} v_{2}$

| joinDb ${ }_{a}^{\text {op }} \quad v_{1} v_{2} v_{3} v_{4}$

Existing forms (Figure 7 )

Open database by file path

Filter DB by predicate

Join DBs using keys' value
\end{abstract}

Figure 10. Database Library Forms

motivating example from Figure 2a. We tour the type system and illustrate how OVV progressively types and validates the operations in this example.

Syntax for libDb. Figure 10 extends the syntax from Figure 7 with the three operations implemented by libDb, for opening databases, filtering them with a predicate, and joining them using named fields. Each operation is parameterized by one or more argument values, and an operation annotation $a^{\text {op }}$ that determines how to type operation. As with record field projection, this annotation determines whether the operation's pre-conditions for success have been fully verified via OVV. The gradual type system for libDb presented here uses different rules for certain (!) versus uncertain (?) reasoning modes.

Types for $\lambda$-VMF and libDb. Figure 11 instantiates the $\lambda$-VMF framework for a gradual type system. This system has (bidirectional, algorithmic) rules to reason about $\lambda$-VMF code as well as the libDb extension. Value types consist of types for thunked computations ( $U$ ) ${ }^{2}$, dictionaries (Dict $\Delta$, where $\Delta$ maps field values to field types), numbers (Num), strings (Str), booleans (Bool), reference cells ( $\operatorname{Ref} A)$, unit (1), unknown (?) and databases (Db $A)$. Computation types consist of the arrow type for

\footnotetext{
2 We follow conventions from the literature on call-by-push-value (CBPV) in our type syntax for thunked and value-returning computations, which uses special letters $\mathbf{U}$ and $\mathbf{F}$, respectively [30,31].
}

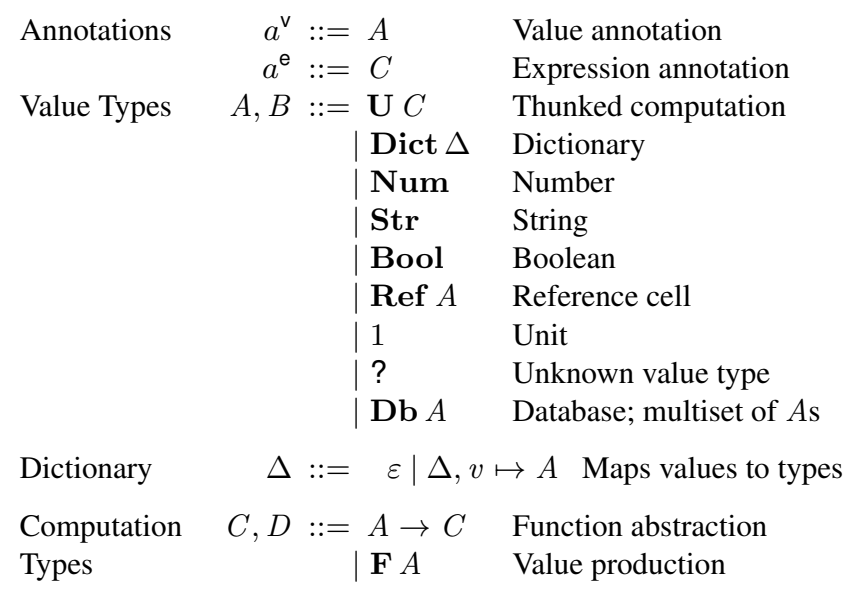

Figure 11. Type Syntax: Annotations for Values and Expressions

functions $(A \rightarrow C)$ and value types for value-producing computations ( $\mathbf{F} A)$.

Typing $\lambda$-VMF program states. Figure 12 lists typing judgement forms for $\lambda$-VMF program states and for stacks. To type a program state, we assume that the stored values are annotated, and we use these annotations as a store typing, written $|\mu|$, which maps reference locations to values types. To type a program state, we assume this store typing $|\mu|$ and attempt to verify the the other VM machinery, consisting of the current environment $\rho$, program term $\dot{e}$, and stack $\kappa$. Three judgements compute type properties for these components: Assuming a store typing $\Gamma$, the judgement $\Gamma \vdash \rho \Rightarrow \Gamma^{\prime}$ computes from an environment (mapping variables to values), a typing context $\Gamma^{\prime}$ (mapping variables to types). Assuming a typing context $\Gamma$, the judgement $\Gamma \vdash e \Rightarrow C$ computes a type from a term $e$. Assuming 
$\sigma$ ok State $\sigma$ is well-typed.

$\frac{|\mu| \vdash \rho \Rightarrow \Gamma \quad|\mu|, \Gamma \vdash \dot{e} \Rightarrow C \quad|\mu| \vdash \kappa \Leftarrow C}{\langle\mu ; \kappa ; \rho ; \dot{e}\rangle \text { ok }}$ state

$\Gamma \vdash \kappa \Leftarrow C$ Under $\Gamma$, stack $\kappa$ eliminates a computation of type $C$.

$$
\begin{aligned}
& \Gamma \vdash \text { halt } \Leftarrow C \text { k-emp } \\
&\left.\frac{\Gamma_{1} \vdash \kappa}{\Gamma_{1} \vdash \kappa::(\rho, x}, e\right) \\
& \Gamma \vdash v \Leftarrow A \\
& \Gamma \vdash \kappa \Leftarrow C \\
& \frac{\Gamma \vdash \kappa:: v \Leftarrow A \rightarrow C}{\Gamma \vdash \text { app }}
\end{aligned}
$$$$
\Gamma_{1} \vdash \rho \Rightarrow \Gamma_{2}
$$$$
\Gamma_{2}, x: A \vdash e \Rightarrow C
$$$$
\frac{\Gamma_{1} \vdash \kappa \Leftarrow C}{\Gamma_{1} \vdash \kappa::(\rho, x . e) \Leftarrow \mathbf{F} A} \text { k-let }
$$

Figure 12. Stack typing and State typing

a typing context $\Gamma$ and computation type $C$ for a terminal computation, the judgement $\Gamma \vdash \kappa \Leftarrow C$ checks that the stack $\kappa$ either correctly continues execution or halts.

The remainder of the figure gives three rules for typechecking the stack. First, k-emp says that halting stacks are always permitted. Next, k-let and k-app handle the recursive cases of the stack, where the topmost frame can be viewed as eliminating the terminal computation type, call it $D$. In the case of k-let, we have that $D$ is $\mathbf{F} A$, which types the terminal computation that returns a value of type $A$; we check that the top of the stack holds the body of the let, which can use the let-bound variable (of type $A$ ), for which we can synthesize another computation type $C$ that checks against the rest of the stack. In the case of k-app, we have that $D$ is $A \rightarrow C$, the type of a function abstraction; we check that the top of the stack is an argument value of type $A$, and the rest of the stack checks against the type of the abstraction's body, $C$.

Transforming $\lambda$-VMF program states. Though the gradual type system defined here is stated propositionally, it constitutes an algorithm, and we demonstrate this fact by implementing these relational definitions as a (mutually) recursive total functions. However, instead of merely returning true or false to indicate the success or failure of the relation to hold, in the case of true, we also construct an annotated term, possibly with transformations (e.g., changing operation annotations from uncertain ? to certain !).

For instance, we implement the type relation for program states as a total function from program states to (optional) program states with annotations; and when the algorithm fails, it returns None: Furthermore, this algorithm plays the role of $e_{\mathrm{m}}$ in libDb's use of $\operatorname{rcc} e_{\mathrm{m}} e$.

The ability to phrase the typing relations as functional algorithms stems the fact that the rules treat certain positions of their (bidirectional) relations consistently as inputs and outputs, and that outputs are determined functionally from inputs. As an example, Figure 6 gives the algorithmic version of the stack-checking relation $\Gamma \vdash \kappa \Leftarrow C$, which resembles an ordinary function in ML. The remainder of the rules transform in a similar manner, so that checking relations produce an optional, annotated term structure, while synthesizing relations produce an optional pair of annotated term structure and synthesized type. When these functions produce None, the corresponding typing relation is not derivable. The dynamic semantics of $\lambda$-VMF do not permit execution to continue when this occurs.

Typing core $\lambda$-VMF terms bidirectionally. For simplicity, we use a bidirectional type system to encode the gradual type systems of the $\lambda$-VMF core calculus and its libDb extension. Figure 13 defines type checking (above) and synthesis (below) for program terms. For space reasons, we elide some synthesis cases, as well as the checking and synthesis judgements for value forms; the rules shown give a representative flavor for the complete definition.

The analytical (checking) judgement form $\Gamma \vdash e \Leftarrow C$ can be read as, "Under typing context $\Gamma$, term $e$ checks against computation type $C$." Specifically, the type $C$ is given as an input to the checking judgement, when viewed as an algorithm. The synthesizing judgement form $\Gamma \vdash e \Rightarrow C$ can be read as, "the typing context $\Gamma$ and term $e$ synthesize the computation type $C$." Specifically, the algorithm computes the type $C$, when given $\Gamma$ and $e$. For the core forms of $\lambda$-VMF, the bidirectional rules for values and computations follow the usual patterns found in bidirectional type systems [10, 16]. We show several standard-looking rules, sub, lam, app and annot. In particular, the annotation form of $\lambda$-VMF, $e$ ?: $a^{\mathrm{e}}$, which asserts the annotation $a^{\mathrm{e}}$ correctly describes the program $e$, plays the role of type ascription in the bidirectional rules; the annot rule says that terms are checked against their type annotations, and these annotated terms synthesize the annotation type. Because it has no dynamic semantics, $\lambda$-VMF uses OVV to prove and discharge this form earlier by rewriting it to $e$ sometime before evaluation; if this rewrite fails, then the program terminates (by failing) early, as a result of OVV failing, not execution.

As is customary in bidirectional systems, type annotations mediate between synthesizing and checking. This provides one the ability to place checking-only terms (such as lambda abstractions) in positions that require the sub-term synthesize a type (such as the abstraction position of an application). See typing rules lam and app, respectively, to see the details; both are standard. Finally, as is customary, type subsumption allows less specific types to check against terms that synthesize more specific types. Rule sub uses a definition of type consistency (written $C \approx$ ? $D$ ), which behaves like type equality, modulo the uncertain type ?, which is consistent with all other types. This notion of consistency is standard in some gradual typing literature [36].

Gradual typing for dictionary projection. The typing rules for uncertain and certain projection differ in what is known 
$\Gamma \vdash e \Leftarrow C$ Under $\Gamma$, expression $e$ checks against type $C$.

$$
\begin{aligned}
& \Gamma \vdash e \Rightarrow C \\
& \frac{C \approx_{?} D}{\Gamma \vdash e \Leftarrow D} \text { sub } \quad \frac{\Gamma, x: A \vdash e \Leftarrow C}{\Gamma \vdash \lambda x . e \Leftarrow A \rightarrow C} \text { lam }
\end{aligned}
$$

$\Gamma \vdash e \Rightarrow C$ Under $\Gamma$, expression $e$ synthesizes type $C$.

$$
\begin{aligned}
& \Gamma \vdash e \Rightarrow A \rightarrow C \\
& \frac{\Gamma \vdash e \Leftarrow C}{\Gamma \vdash e ?: C \Rightarrow C} \text { annot } \quad \frac{\Gamma \vdash v \Leftarrow A}{\Gamma \vdash e v \Rightarrow C} \text { app } \\
& \Gamma \vdash v_{1} \Rightarrow ? \quad \Gamma \vdash v_{1} \Rightarrow \operatorname{Dict}\left(\Delta, v_{2} \mapsto A\right) \\
& \frac{\Gamma \vdash v_{2} \Rightarrow B}{\Gamma \vdash v_{1}\left[v_{2}\right]_{?} \Rightarrow \mathbf{F} ?} \mathrm{p} \text { ? } \\
& \begin{array}{r}
\Gamma \vdash v_{2} \Rightarrow B \\
\vdash v_{1}\left[v_{2}\right]_{!} \Rightarrow \mathbf{F} A
\end{array} \\
& \frac{\Gamma \vdash v \Rightarrow \mathbf{S t r}}{\Gamma \vdash \text { openDb }_{?} v \Rightarrow \mathbf{F}(\mathbf{D b} ?)} \text { openDb? } \\
& \Gamma \vdash v_{1} \Rightarrow \mathbf{D b} \text { ? } \\
& \frac{\Gamma \vdash v_{2} \Leftarrow \mathbf{U}(? \rightarrow \mathbf{F} \text { Bool })}{\Gamma \vdash \text { filterDb? } v_{1} v_{2} \Rightarrow \mathbf{F}(\mathbf{D b} ?)} \text { filterDb? } \\
& \Gamma \vdash v_{1} \Rightarrow \mathbf{D b} A \quad ? \notin A \\
& \frac{\Gamma \vdash v_{2} \Leftarrow \mathbf{U}(A \rightarrow \mathbf{F} \text { Bool })}{\Gamma \vdash \text { filterDb! } v_{1} v_{2} \Rightarrow \mathbf{F}(\mathbf{D b} A)} \text { filterDb! } \\
& \Gamma \vdash v_{1} \Rightarrow \mathbf{D b} ? \quad \Gamma \vdash v_{2} \Rightarrow B_{2} \\
& \Gamma \vdash v_{3} \Rightarrow \mathbf{D b} ? \quad \Gamma \vdash v_{4} \Rightarrow B_{4} \\
& \overline{\Gamma \vdash \text { joinDb}_{\text {? }} v_{1} v_{2} v_{3} v_{4} \Rightarrow \mathbf{F}(\mathbf{D b} ?)} \text { joinDb? } \\
& \Delta=\Delta_{1}, v_{2} \mapsto A, \Delta_{3}, v_{4} \mapsto A \\
& \Gamma \vdash v_{1} \Rightarrow \mathbf{D b}\left(\boldsymbol{D i c t}\left(\Delta_{1}, v_{2} \mapsto A\right)\right) \quad \Gamma \vdash v_{2} \Rightarrow B_{2} \\
& \frac{\Gamma \vdash v_{3} \Rightarrow \mathbf{D b}\left(\boldsymbol{D i c t}\left(\Delta_{3}, v_{4} \mapsto A\right)\right) \quad \Gamma \vdash v_{4} \Rightarrow B_{4}}{\Gamma \vdash \text { joinDb } v_{1} v_{2} v_{3} v_{4}: \mathbf{F}(\mathbf{D b}(\mathbf{D i c t} \Delta))} \text { joinDb! }
\end{aligned}
$$

\begin{tabular}{|c|c|c|c|c|}
\hline & $\begin{array}{cc}\text { Line 1 } & \text { Line 2 } \\
\text { openDb } & \text { filterDb }\end{array}$ & $\begin{array}{c}\text { Line } 3 \\
\text { openDb }\end{array}$ & $\begin{array}{l}\text { Line } 4 \\
\text { joinDb }\end{array}$ & $\cdots$ \\
\hline 1: & $?$ & $?$ & $?$ & $?$ \\
\hline$\overline{2}: \overline{f i}$ & - & $\bar{?}$ & $?$ & $\overline{!} \bar{\ell}$ \\
\hline 3: ol & 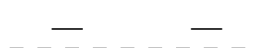 & $?$ & ? & $! / ?$ \\
\hline$\overline{4}: \overline{\text { joinD }} \overline{\mathrm{D}} \overline{\mathrm{b}}$ & - & - & $!$ & $\overline{!}$ \\
\hline
\end{tabular}

Figure 13. Selected typing rules for computation typing (checking and synthesis).

Typing (horizontal) across Execution (vertical).

Figure 14. Progressive typing for Figure 2a As execution progresses (vertically) over Lines 1 and 3, the continuation's typing becomes more certain: After Line 1 executes, Line 2 types in the certain modality (!) instead of the uncertain modality (?); similarly, after Line 3, Lines 4 onward type using the certain modality. (The dashed horizontal lines indicate these progressions). about the record and field values, and illustrate a form of gradual typing. In the uncertain case, rule p? synthesizes return type ?, since nothing is known about the dictionary of values being projected. By contrast, in the certain case, the dictionary type is known to rule p!, and this dictionary maps the given field value to a corresponding field type. In this case, the soundness of the type system means that the projection must succeed in all possible future program states, and moreover, that the projected value has the given type.

Typing the libDb operations. The rule openDb? is uncertain and has no certain counterpart: The type of the database is not known until after the operation completes, just before execution resumes with its continuation; before then, the database could hold any type, so the rule types the returned database as $\mathbf{D b}$ ?.

Following similar reasoning, since filtering and joining databases occur after a database is loaded, it is possible to type these operations in both uncertain and certain modes. The rule filterDb? says that filtering a database of uncertain values leads to another database of values with an uncertain type; since it merely assumes the type of the database is ?, it does not prove that the predicate will not "go wrong", e.g., by projecting the wrong field from its argument. By contrast, the rule filterDb! says that filtering a database of known type using a predicate that checks against this type leads to a database with the same known type; in this case, the soundness of the type system means that the predicate must always succeed.

Similarly, the rules for joinDb? and joinDb! follow the pattern set above: the uncertain rule assumes nothing about the argument values, beyond the arguments actually consisting of databases. The certain rule assumes that the database arguments' types are fully known, that the chosen field values are mapped in these types, and that the chosen fields share a common type (we want to compare values of this field for equality to perform the join).

Gradual Typing, Progressively via OVV. Figure 14 illustrates using the typing rules of Figure 13 to perform progressive typing our four-line motivating example (Figure 2a). The vertical and horizontal dimensions of the table list each of the four lines; the vertical axis represents concrete execution, and the horizontal axis represents typing the four right-hand-sides of the program's four let-bindings, and in particular, for filterDb (on Line 2) and joinDb (on Line 4), the table indicates whether the operation was typed in the uncertain or certain modality. As execution progresses (vertically) over Lines 1 and 3, the typing of the program continuation's becomes more certain: After Line 1 executes, Line 2 types in the certain modality (!) instead of the uncertain modality (?); similarly, after Line 3, Lines 4 onward type using the certain modality. As the final column shows, the certainty of code using these tables in the remainder of the program (Line 5 onwards) increases after each of the two calls to openDb. Between the two calls, some information is 


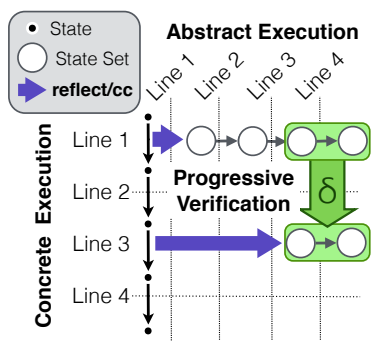

(a) Progressive verification

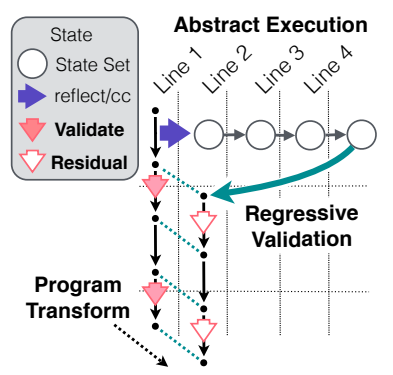

(b) Regressive validation
Figure 15. Progressive verification and regressive validation are complementary ways of seeing the interplay between $\forall$-analysis and $\exists$-analysis enabled by online verification-validation.

known (relating to the first two tables defined on Lines 1 and 2), but some information is still missing (relating to the two tables defined in Lines 3 and 4).

Our current Rust-based prototype of $\lambda$-VMF is powerful enough to express this example, including the progressive typing discussed above. In Section 5 , we discuss the potential to use incremental computation in the context of such progressive typing; the goal is to improve performance by exploiting the redundancy of re-typing the program's continuation.

\section{Discussion}

In this section, we discuss future challenges and directions for the vision of OVV presented in this paper. Specifically, we discuss the design of the meta-level programming language, and its use in expressing progressive verification and regressive validation.

Incremental Computation for Progressive Verification. In the motivating example from Section 2, the chk_stk calls performed by openDb use progressive typing to check their continuations. In fact, these two continuations are related: The earlier version lacks type information about the table loaded in line 3, whereas the later version has access to this type information. Progressive typing could exploit this incremental relationship to avoid re-computing all of the typing facts about the program state that have not changed.

Progressive typing is a specific instance of progressive verification. Pictorially, progressive verification relates distinct abstract executions (shown horizontally in Figure 15a, by exploiting their similarity (a small change, depicted as $\delta$, extending vertically). The task of a progressive verifier is a $\forall$-analysis, just like a classical static verifier, which attempts to prove that all executions to an assertion satisfy a particular safety property.

More Aggressive Regressive Validation. After type checking the continuation for line 1 , regressive validation con- sists of eliminating dynamic checks within the library calls of lines 2 and 4 (filterDb and joinDb, respectively). In particular, if the continuation type-checks under the partiallyknown type information, the known type information can be used to elide run-time $\exists$-analysis checks that concern the authors table, including the asserts for the projections of author.name. Pictorially, we think of regressive validation as introducing a third dimension that consists of all possible outcomes of a program transformation on the object program (shown in Figure 15b. After performing an online verification, the meta layer transforms the continuation, either eliding certain downstream validation checks (labeled validate), or introducing residual checks that reduce the original checks' complexity (labeled residual). As illustrated in Section 4 progressive typing can, before executing the operation, eliminate residual checks by rewriting uncertain operations to certain operations (which require no run-time type checks). More aggressive verification techniques can hope to regress even more aggressive validation checks to simpler forms. For instance, global heap-based properties present an interesting challenge.

Implicitly-Incremental Meta-Level Computation. In sum, the example chk_stk above encodes a theory about online typing, along with a mechanism for using this type information to optimize the dynamic run-time checks that would otherwise be used. The meta layer should have an in-built ability to implicitly express progressive verification as ordinary verification, so that the system, not the programmer, takes into account execution environment changes across these progressive stages. Further, when the meta layer uses this progressive verification to enable regressive validation in the future execution down stream, the system, not the programmer, accounts for these changes when doing future stages of progressive verification. In other words, the VM that runs the meta language and object language should have an in-built ability to express interaction among the levels in terms of implicitly-incremental computation. In Section 6, we discuss the challenges that OVV poses to work on general-purpose incremental computation.

\section{Related Work}

This section supplements the related work in Section 2 .

General-Purpose Incremental Computation. Section 5 proposes an implicitly incremental meta-level language for $\lambda$-VMF, which challenges current research on (generalpurpose, programming language-based) incremental computation (IC). Consider the desired incremental behavior of chk_stk in the motivating example, where it occurs after lines 1 and 3, when the program states are similar, but not identical. In particular, both continuations include the AST of the call in line 4, and onward, which chk_stk will process in both verification stages. The central challenge is reusing the redundant work performed by chk_stk, despite the fact 
that the AST and store typing are not equal to that in the prior stage, which creates challenges for incremental computing via memoization, a key implementation mechanism used across many specific IC approaches.

To understand why these "small" changes are challenging for typical memoization, consider the structural recursion of the chk_stk function from Figure 6. One approach to memoization identifies each saved invocation by the entire store typing st and entire stack stk, including all of their recursive sub-structure, e.g., via hash-consing [18]. This approach is commonly taken by past work on incremental computation [7, 8, 17, 24, 25, 33, 34, 34], however, it is brittle and overly sensitive to small changes, since they alter the identity of the whole recursive structure. Recent work addresses this shortcoming by introducing unique names that are special to incremental computing [26]. This naming mechanism can overcome the challenges outlined above for chk_stk, since the presence of names isolates changed components of the store typing, stack and local environments.

However, several key challenges remain before these techniques can fully realize OVV: We want to use these names correctly (to avoid unsound incremental results), use them efficiently (to isolate changes and avoid sub-redundant computations) and use them implicitly (so that the meta-level programs look like ML). Further, we may want to control how fine-grained the IC techniques track program dependencies, to reduce constant-factor overhead.

Reflective Towers of Interpreters. As proposed in Section 5 future work on $\lambda$-VMF should permit library extension authors to write meta programs and object programs in an integrated way. Fortunately, many researchers have proposed designs that allow interesting interplay between the interpreter's viewpoint (where the meta-level program runs) and the program being interpreted (where the object program runs). Conceptually, this work begins with 3-LISP [14, 38], which gives the programmer access to an infinite tower of (so-called meta-circular) interpreters, allowing them to redefine the language from within the language. Following (theoretical) work on 3-LISP, researchers give various approaches that attack practical concerns in how to express and implement reflective towers in simpler terms; these efforts are named after various hair colors: Brown [19, 40], Blond [13] and most recently, Black [3, 4].

Compared to the impressive and mind-bending work on metacircular interpreters, the vision for $\lambda$-VMF is more modest: Two levels suffice to perform OVV. Having said that, if meta-level programmers want to verify their metalevel programs as object programs (to "bootstrap" a typed meta level), the work mentioned above will likely provide further insights.

Program Analysis for Dynamic Languages. The ultimate aim of online verification-validation is offer "strong checking" in an extensible, dynamic language environment. And thus we seek to build on the substantial amount of research activity on program analysis for dynamic languages. Since by definition, dynamic languages lack a built-in static typing discipline, much of the static verification work focuses on either retrofitting rich typing or specification disciplines [11, 12, 21 or applying whole-program flow analysis for inferring and checking type properties (e.g., for JavaScript [5, 27 28] or for Ruby [1]). Researchers have also used static type inference to instrument dynamic programs with assertions that fail preemptively [23]; while similar in spirit to OVV, such approaches are still phasic, since type analysis occurs only once, before program execution begins.

The dynamic language features that make widely-used libraries like jQuery possible also make retrofitting static techniques incredibly challenging [2, 29, 35, 39]. Much of this work focuses on finding the right kinds of context-sensitivity to try to more precisely resolve the flow of values to dynamic features like dynamic property read in a static analysis [2, 32, 39] or to determine when dynamically-observed information is sufficient to apply in a static verification [35]. We expect such techniques to be not only applicable and useful but strengthened in an OVV context. In the end, static techniques in a phasic setting are limited by what is indeed available statically, and dynamic techniques are limited by what can be observed in testing runs. As exhibited in Section 2, the vision of OVV enables these techniques to be strengthened with a flexible interleaving of "static" and "dynamic" analysis (i.e., $\forall$-analysis and $\exists$-analysis).

\section{Conclusion}

This paper presents a vision for online verificationvalidation (OVV), an approach to ease the tension between extensibility (of dynamic languages) and safety (of static languages). The key insight of OVV is that analysis in a VM can be phaseless, allowing analyses to run progressively on the object program by pausing execution, reflecting on the current continuation, and transforming the continuation to replace uncertain (?) operations with certain (!) ones.

In this paper, we formalize an approach for OVV as a language semantics and Rust-based implementation called $\lambda$ VMF. We explore a proof-of-concept instantiation of OVV by defining a gradual type system for dynamic field projection and databases with dynamic schemas, and we observed that the result is a progressive type checker.

\section{Acknowledgments}

This material is based in part upon work supported by a gift from Mozilla, and support from the National Science Foundation under grant numbers CCF-1619282, CCF1618756, CCF-1055066 and CCF-1218208. Any opinions, findings, and conclusions or recommendations expressed in this material are those of the author(s) and do not necessarily reflect the views of Mozilla, or the National Science Foundation. 


\section{References}

[1] Jong-hoon (David) An, Avik Chaudhuri, and Jeffrey S. Foster. Static typing for Ruby on Rails. In Automated Software Engineering (ASE), 2009.

[2] Esben Andreasen and Anders Møller. Determinacy in static analysis for jQuery. In Object-Oriented Programming Systems, Languages, and Applications (OOPSLA), 2014.

[3] Kenichi Asai. Reflection in direct style. In Generative Programming and Component Engineering (GPCE), 2011.

[4] Kenichi Asai, Satoshi Matsuoka, and Akinori Yonezawa. Duplication and partial evaluation for a better understanding of reflective languages. Lisp and Symbolic Computation, 9(2-3), 1996.

[5] SungGyeong Bae, Hyunghun Cho, Inho Lim, and Sukyoung Ryu. SAFEWAPI: web API misuse detector for web applications. In Foundations of Software Engineering (FSE), 2014.

[6] Nels E. Beckman, Aditya V. Nori, Sriram K. Rajamani, Robert J. Simmons, Sai Deep Tetali, and Aditya V. Thakur. Proofs from tests. IEEE Transactions on Software Engineering, 2010.

[7] Pramod Bhatotia, Alexander Wieder, Rodrigo Rodrigues, Umut A. Acar, and Rafael Pasquin. Incoop: MapReduce for incremental computations. In Cloud Computing (SoCC), 2011.

[8] Pramod Bhatotia, Pedro Fonseca, Umut A. Acar, Björn B. Brandenburg, and Rodrigo Rodrigues. iThreads: A threading library for parallel incremental computation. In Conference on Architectural Support for Programming Languages and Operating Systems (ASPLOS), 2015.

[9] Eric Bodden, Andreas Sewe, Jan Sinschek, Hela Oueslati, and Mira Mezini. Taming reflection: Aiding static analysis in the presence of reflection and custom class loaders. In International Conference on Software Engineering (ICSE), 2011.

[10] Adam Chlipala, Leaf Petersen, and Robert Harper. Strict bidirectional type checking. 2005.

[11] Ravi Chugh, David Herman, and Ranjit Jhala. Dependent types for JavaScript. In Object-Oriented Programming Systems, Languages, and Applications (OOPSLA), 2012.

[12] Ravi Chugh, Patrick Maxim Rondon, and Ranjit Jhala. Nested refinements: a logic for duck typing. In Principles of Programming Languages (POPL), 2012.

[13] Olivier Danvy and Karoline Malmkjær. Intensions and extensions in a reflective tower. In LISP and Functional Programming, 1988.

[14] Jim des Rivières and Brian Cantwell Smith. The implementation of procedurally reflective languages. In LISP and Functional Programming, 1984.

[15] Bruno Dufour, Barbara G. Ryder, and Gary Sevitsky. Blended analysis for performance understanding of framework-based applications. In Software Testing and Analysis (ISSTA), 2007.

[16] Joshua Dunfield and Neelakantan R. Krishnaswami. Sound and complete bidirectional typechecking for higher-rank poly- morphism with existentials and indexed types. CoRR, abs/1601.05106, 2016.

[17] Sebastian Erdweg, Oliver Bracevac, Edlira Kuci, Matthias Krebs, and Mira Mezini. A co-contextual formulation of type rules and its application to incremental type checking. In Object-Oriented Programming Systems, Languages, and Applications (OOPSLA), 2015.

[18] Jean-Christophe Filliâtre and Sylvain Conchon. Type-safe modular hash-consing. In Proceedings of the 2006 Workshop on ML. ACM, 2006.

[19] Daniel P. Friedman and Mitchell Wand. Reification: Reflection without metaphysics. In LISP and Functional Programming, 1984.

[20] Michael Furr, Jong hoon (David) An, and Jeffrey S. Foster. Profile-guided static typing for dynamic scripting languages. In Object-Oriented Programming Systems, Languages, and Applications (OOPSLA), 2009.

[21] Philippa Gardner, Sergio Maffeis, and Gareth David Smith. Towards a program logic for JavaScript. In Principles of Programming Languages (POPL), 2012.

[22] Patrice Godefroid, Nils Klarlund, and Koushik Sen. DART: Directed automated random testing. In Programming Language Design and Implementation (PLDI), 2005.

[23] Neville Grech, Julian Rathke, and Bernd Fischer. Preemptive type checking in dynamically typed languages. In Theoretical Aspects of Computing - ICTAC, 2013.

[24] Philip J. Guo and Dawson Engler. Using automatic persistent memoization to facilitate data analysis scripting. In Software Testing and Analysis (ISSTA), 2011.

[25] Matthew A. Hammer, Yit Phang Khoo, Michael Hicks, and Jeffrey S. Foster. Adapton: Composable, demand-driven incremental computation. In Programming Language Design and Implementation (PLDI), 2014.

[26] Matthew A. Hammer, Joshua Dunfield, Kyle Headley, Nicholas Labich, Jeffrey S. Foster, Michael W. Hicks, and David Van Horn. Incremental computation with names. In Object-Oriented Programming Systems, Languages, and Applications (OOPSLA), 2015.

[27] Simon Holm Jensen, Anders Møller, and Peter Thiemann. Type analysis for JavaScript. In Static Analysis (SAS), 2009.

[28] Vineeth Kashyap, Kyle Dewey, Ethan A. Kuefner, John Wagner, Kevin Gibbons, John Sarracino, Ben Wiedermann, and Ben Hardekopf. JSAI: a static analysis platform for JavaScript. In Foundations of Software Engineering (FSE), 2014.

[29] Benjamin S. Lerner, Liam Elberty, Jincheng Li, and Shriram Krishnamurthi. Combining form and function: Static types for jQuery programs. In Object-Oriented Programming (ECOOP), 2013.

[30] Paul Blain Levy. Call-by-push-value: A subsuming paradigm. In Typed Lambda Calculi and Applications (TLCA), 1999.

[31] Paul Blain Levy. Call-by-push-value: A Functional/imperative Synthesis, volume 2. 2003.

[32] Changhee Park and Sukyoung Ryu. Scalable and precise static analysis of JavaScript applications via loop-sensitivity. In 
Object-Oriented Programming (ECOOP), 2015.

[33] William Pugh. Incremental Computation via Function Caching. PhD thesis, Cornell University, 1988.

[34] William Pugh and Tim Teitelbaum. Incremental computation via function caching. In Principles of Programming Languages (POPL), 1989.

[35] Max Schäfer, Manu Sridharan, Julian Dolby, and Frank Tip. Dynamic determinacy analysis. In Programming Language Design and Implementation (PLDI), 2013.

[36] Jeremy G. Siek and Walid Taha. Gradual typing for functional languages. In Scheme and Functional Programming Workshop, 2006.

[37] Jeremy G. Siek and Walid Taha. Gradual typing for objects. In Object-Oriented Programming (ECOOP), 2007.
[38] Brian Cantwell Smith. Reflection and semantics in Lisp. In Principles of Programming Languages (POPL), 1984.

[39] Manu Sridharan, Julian Dolby, Satish Chandra, Max Schäfer, and Frank Tip. Correlation tracking for points-to analysis of JavaScript. In Object-Oriented Programming (ECOOP), 2012.

[40] Mitchell Wand and Daniel P. Friedman. The mystery of the tower revealed: A nonreflective description of the reflective tower. Lisp and Symbolic Computation, 1(1), 1988.

[41] Shiyi Wei and Barbara G. Ryder. Practical blended taint analysis for JavaScript. In Software Testing and Analysis (ISSTA), 2013. 